\title{
Características clínicas e da saturação transcutânea de oxigênio em lactentes hospitalizados com bronquiolite viral aguda
}

\author{
Clinical and transcutaneous oxygen saturation characteristics \\ in hospitalized infants with acute viral bronchiolitis
}

\author{
Fernanda M. Rubin'1 , Gilberto B. Fischer ${ }^{2}$
}

\begin{abstract}
Resumo
Objetivos: Descrever as características clínicas de lactentes com bronquiolite viral aguda (BVA) e verificar a influência do tempo de dessaturação de oxigênio (TD) como teste preditivo para estimar a evolução desses pacientes.

Métodos: Estudo de coorte realizado com 111 lactentes hospitalizados com diagnóstico de BVA e em oxigenoterapia por cateter nasal. Os desfechos foram: tempo de internação, tempo de oxigenoterapia e tempo para saturar $95 \% \mathrm{em}$ ar ambiente. Foi aplicado, duas vezes ao dia, um escore de gravidade com os sinais clínicos verificados durante o período em que o paciente necessitou de oxigênio suplementar. Após suspender o oxigênio do paciente, foi observado o tempo necessário para a saturação transcutânea de oxigênio decrescer até $90 \%$ e $85 \%$. Utilizou-se o teste do qui-quadrado ou teste exato de Fisher, para comparar entre si os grupos de variáveis categóricas, e o teste $t$ ou Mann-Whitney, para as variáveis numéricas. A correlação de Spearman foi utilizada para avaliar associações entre variáveis contínuas de distribuição assimétrica.
\end{abstract}

Resultados: Houve predominância de idade inferior a quatro meses $(61,3 \%)$. Os pacientes com história de sibilância prévia (45\%) foram analisados separadamente e tiveram resultados semelhantes ao grupo com BVA ( $\mathrm{p}=0,05)$. A prevalência de desnutrição moderada e grave foi de 26 pacientes (23\%). Todos os pacientes utilizaram broncodilatador; $20 \%$ receberam corticosteróides sistêmicos e $42,7 \%$, antibióticos. A mediana do tempo de oxigenoterapia até saturar $95 \%$ em ar ambiente foi de 83 horas (IIQ 55-128). A mediana do tempo de internação hospitalar foi de 7 dias (IIQ 5-10,5). Observou-se pouca variabilidade clínica no período estudado. Não se encontraram correlações estatisticamente significantes $(\mathrm{p}=0,002)$ entre os escores clínicos e os TDs com os desfechos.

Conclusões: Os TDs como elementos auxiliares na avaliação de pacientes em oxigenoterapia não foram clinicamente úteis neste estudo. É possível, no entanto, que se verifique melhores resultados utilizando-se esta medida em pacientes com maior variabilidade clínica.

J Pediatr (Rio J) 2003;79(5):435-42: Bronquiolite, terapia, complicações.

\begin{abstract}
Objective: To describe the clinical characteristics of infants with acute viral bronchiolitis (AVB) and to assess the influence of oxygen desaturation time (DT) as a prognostic test to estimate the evolution of such patients.
\end{abstract}

Methods: We performed a cohort study with 111 hospitalized patients diagnosed with AVB receiving oxygen therapy through nasal prong. The outcomes were: length of admission, length of oxygen therapy and time elapsed to read $95 \%$ saturation in room air. A severity score was obtained twice a day based on clinical signs during the time when the patient required oxygen supplementation. After the supply of oxygen was interrupted, the time required for transcutaneous oxygen saturation decreased to $90 \%$ and $85 \%$. The $\chi^{2}$ test or Fisher's exact test were used to compare categorical variables. The $t$ test or Mann-Whitney's test were used for numerical variables. Spearman's correlation was used to evaluate associations in continuous variables with asymmetric distribution.

Results: Most patients (61.3\%) were younger than 4 months. Patients with wheezing history (45\%) were analyzed separately and had similar results to those of the group with AVB $(\mathrm{p}=0.05)$. Twentysix patients $(23 \%)$ had moderate or severe malnutrition. All patients were using bronchodilators; $20 \%$ were using systemic corticosteroids; and $47 \%$, antibiotics. The median time of oxygen therapy required for a reading of $95 \%$ oxygen saturation in room air was 83 hours (IQI 55128). The median of length of admission was 7 days (IQI 5-10.5). Little clinical variability was observed in the period studied. No significant correlations were found between the clinical scores, DT and the outcomes.

Conclusion: DT was not useful as an aid to assess AVB patients on oxygen therapy in this study. It is possible that this tool could have been more useful in patients with more clinical variability.

J Pediatr (Rio J) 2003;79(5):435-42: Bronchiolitis, therapy, complications.

1. Pediatra intensivista, mestranda em Pediatria pela Universidade Federal do Rio Grande do Sul - UFRGS.

2. Doutor em Pneumologia, Professor titular de Pediatria da Fundação Faculdade Federal de Ciências Médicas de Porto Alegre - FFFCMPA

Auxílio financeiro: Bolsa de Iniciação Científica FAPERGS.

Artigo submetido em 02.01.02, aceito em 28.05.03 


\section{Introdução}

A bronquiolite viral aguda (BVA) é uma das causas mais comuns de infecção das vias aéreas inferiores em crianças abaixo de um ano de idade e determina um expressivo número de hospitalizações ${ }^{1}$.

O diagnóstico de BVA é principalmente clínico. A doença se caracteriza como primeiro episódio de uma doença das vias aéreas inferiores em crianças com idade inferior a 12 meses, precedida por um período prodrômico de três a cinco dias com sinais de infecção de vias aéreas superiores, com coriza, tosse e febre, que evolui nos dias subseqüentes com taquipnéia, tosse, sibilos e sinais de dificuldade ventilatória ${ }^{2,3}$. É uma doença geralmente benigna e autolimitada, que, no entanto, produz morbidade significativa em lactentes pequenos ${ }^{4}$ e pacientes portadores de doenças crônicas ${ }^{4-9}$, e freqüentemente causa sintomatologias ventilatórias em longo prazo ${ }^{10}$.

O entendimento das condições que determinam a evolução da BVA pode auxiliar na previsão das necessidades de recursos que devem ser disponibilizados para o adequado tratamento. A oxigenoterapia é o recurso terapêutico amplamente aceito nessa doença ${ }^{11}$, sendo a monitorização por oximetria de pulso a avaliação mais prática para adequá-la às necessidades de cada paciente ${ }^{12}$. Considerando que a saturação transcutânea de oxigênio da hemoglobina (SatHb) é uma medida fidedigna da oxigenação ${ }^{13,14}$, o tempo em que ocorre a queda desta medida ao ser retirado o oxigênio (tempo de dessaturação $=\mathrm{TD}$ ) poderia expressar a maior necessidade dessa terapêutica. Potencialmente, o TD poderia acrescentar informações de caráter prognóstico em pacientes com BVA. Assim, procurou-se descrever as características clínicas de lactentes hospitalizados com diagnóstico com BVA nos primeiros dias de internação, além de verificar se o TD tem fator prognóstico em pacientes hospitalizados por BVA. A gravidade foi verificada através do tempo de internação, do tempo de oxigenoterapia e do tempo para saturar $95 \%$ em ar ambiente.

\section{Pacientes e métodos}

Durante o período de maio a outubro de 2001, realizouse um estudo de coorte que incluiu 111 pacientes entre $1 \mathrm{e}$ 12 meses de idade, internados no Hospital da Criança Santo Antônio, de Porto Alegre, com diagnóstico de BVA estabelecido pelo médico assistente na admissão, $\mathrm{SatHb}$ menor do que $95 \%$ e em oxigenoterapia por cateter extranasal há menos de 24 horas. Entre esses 111 pacientes, aqueles que não completavam os critérios para o diagnóstico de $\mathrm{BVA}^{2}$, após avaliação detalhada para inclusão no estudo, por apresentarem história de sibilância prévia, foram analisados separadamente.

As crianças com doenças cardiocirculatórias (como, por exemplo, doença cardíaca congênita cianótica), doenças neuromusculares (tais como paralisia cerebral e doença de Werdnig-Hoffmann) ou outras (como por exemplo doenças metabólitas congênitas) que afetassem a função ventilatória foram excluídas. Cerca de $70 \%(\mathrm{n}=194)$ das exclusões se deram em razão de o lactente apresentar SatHb maior ou igual a $95 \%$ em ar ambiente.

Foram realizadas avaliações clínicas duas vezes ao dia (manhã e tarde), durante o período em que o paciente necessitou de oxigênio suplementar (até atingir $\mathrm{SatHb}$ 95\% em ar ambiente), com limite de dez avaliações. Os pacientes incluídos tiveram o oxigênio adicional retirado, mantendo-se a aferição da SatHb. Foi verificado, então, o tempo decorrido para a SatHb decrescer até 90\% (TD90) e 85\% (TD85), limitando-se a medida a, no máximo, 5 minutos.

Para a avaliação da saturação, foram utilizados oxímetros de pulso das marcas Omehda, modelo 3800, e MiniScope II - Instramed. A pesquisa de vírus respiratório por imunofluorescência indireta (fabricante Chenicon - Respiratory Panel I Viral Screening The Identification Kit) foi feita a partir da obtenção de uma amostra de secreção nasofaríngea, mediante aspiração com sonda número 6 ou 8 , conforme a rotina do hospital.

Com os sinais clínicos anotados, foi constituído um escore de gravidade, adaptado de Fischer ${ }^{15}$. A escala incluiu frequiência respiratória ( $>60$ movimentos por minuto $=1$ ), batimento de asa do nariz (presente $=1$ ), tiragem subcostal $($ presente $=1)$, tiragem intercostal $($ presente $=1)$, tiragem supra-esternal (presente $=1$ ), cianose de extremidades (presente $=1$ ) e compressão do leito ungueal com enchimento capilar lento (presente $=1$ ). $\mathrm{Na}$ ausência do sintoma ou frequiência respiratória menor ou igual a 60 movimentos por minuto, não foi registrada pontuação. $\mathrm{O}$ valor máximo foi 7 , e o valor mínimo, zero.

O cálculo do tamanho da amostra foi feito a partir de um estudo piloto realizado com 20 pacientes. Para correlações entre os TDs e escores com os desfechos, encontrou-se que, para alfa de 0,5 e poder de $95 \%$, seriam necessários 20 pacientes com dados completos para coeficiente de correlação (r) de 0,7 .

A análise estatística foi realizada através dos programas SPSS (Statistical Package for Social Sciences), versão 6.0, e Epi-Info 6, versão 6.04d.

Inicialmente, foram descritas as características gerais e estratificadas por episódio prévio de sibilância: foram realizadas tabelas de frequiências para as variáveis categóricas e calculadas as médias (e desvio padrão) ou medianas (e intervalo interquartis 25-75) para as variáveis contínuas, para as quais foram elaborados diagramas de caixas.

Utilizou-se o teste do qui-quadrado ou teste exato de Fisher, para comparar entre si os grupos de variáveis categóricas, e o teste $t$ ou Mann-Whitney, para as variáveis numéricas. A correlação de Spearman foi utilizada para avaliar associações entre variáveis contínuas de distribuição assimétrica (escore de gravidade, tempo de internação, 
tempo de oxigenoterapia total e tempo de oxigenoterapia até saturar acima ou igual a 95\% em ar ambiente). Considerou-se alfa crítico de 5\% em todas as comparações, exceto nas correlações para comparações múltiplas, em que foi utilizada a correção de Bonferroni (30 correlações: $\mathrm{p}=0,002 ; 10$ correlações: $\mathrm{p}=0,005$ ).

Os dados relativos ao peso e estatura para a idade foram digitados e analisados no programa específico do Epi-Info que utiliza o padrão NCHS (EpiNut) ${ }^{16}$.

O projeto foi submetido ao comitê de ética do Complexo Hospitalar Santa Casa de Porto Alegre e ao Grupo de Pesquisa e Pós-graduação do Hospital de Clínicas de Porto Alegre, tendo recebido aprovação em ambos. Foi solicitada autorização aos responsáveis dos pacientes através de um termo de consentimento informado. A equipe da pesquisa não interferiu na prescrição dos pacientes.

\section{Resultados}

Foram incluídos 111 pacientes. Houve leve predomínio do sexo masculino (54\%) e freqüência elevada de idade inferior a quatro meses $(n=68 ; 61,3 \%)$. Os meses mais prevalentes foram junho e julho $(n=52 ; 47 \%)$.

Os antecedentes mórbidos foram caracterizados pelas variáveis apresentadas na Tabela 1. Em relação aos diagnósticos prévios à internação, observou-se que $21 \%$ dos pacientes tinham história de BVA, $14 \%$ de pneumonia, $4 \%$ de asma e $3 \%$ de bronquite.

As manifestações respiratórias prévias à hospitalização, referidas pelos familiares, ocorreram na sua maioria nos três dias anteriores à internação: "falta de ar" em 64\% $(\mathrm{n}=101)$, "chiado no peito" em 55\% ( $\mathrm{n}=104)$, "febre" em $72 \%(\mathrm{n}=74)$ e parar de respirar em $94 \%(\mathrm{n}=16)$.

Em relação à comparação das características dos pacientes com história prévia de sibilância com às daqueles sem esse antecedente, não se observaram diferenças significativas entre os grupos, em relação ao sexo, prematuridade, necessidade de ventilação mecânica prévia, peso de nascimento, peso e estatura atuais e tempo de internação $(p=0,05)$.

Quanto ao estado nutricional, excluídos os pacientes com história de prematuridade, 26 pacientes (23\%) apresentavam desnutrição moderada ou grave.

Dentre as complicações infecciosas diagnosticadas, observou-se que 23 pacientes $(20,7 \%)$ apresentavam pneumonia, $6(5,4 \%)$ otite média aguda, e $2(1,8 \%)$ septicemia. Observou-se que 29 pacientes $(26,1 \%)$ tinham um ou mais diagnósticos de infecções bacterianas associadas.

Apesar de o diagnóstico inicial dos pacientes ser de bronquiolite viral aguda, durante a internação, outros rótulos diagnósticos foram definidos, tais como: broncospasmo $(\mathrm{n}=29 ; 26,1 \%)$, displasia broncopulmonar $(\mathrm{n}=1$; $0,9 \%)$, asma $(\mathrm{n}=4 ; 3,6 \%)$, lactente sibilante $(\mathrm{n}=2 ; 1,8 \%)$ e bronquiolopatia pós-viral $(\mathrm{n}=1 ; 0,9 \%)$.

Todos os pacientes utilizaram nebulização com broncodilatador (fenoterol) e somente uma criança recebeu broncodilatador endovenoso (salbutamol). Houve uso de corticosteróide sistêmico em cerca de metade da população estudada ( $\mathrm{n}=59 ; 53,2 \%)$, a maioria com história de sibilância prévia $(\mathrm{n}=39 ; 78 \%)$. O uso de antibiótico sistêmico endovenoso, observado em 52 crianças $(46,8 \%)$, esteve distribuído uniformemente em relação à sibilância prévia ( $\mathrm{n}=26 \mathrm{em}$ cada grupo): $52 \%$ nos pacientes com antecedente de sibilância e $42,6 \%$ nos com primeiro episódio ( $\mathrm{p}=0,427)$.

Em relação à identificação etiológica viral, ocorreram 77 resultados negativos $(69,4 \%)$, e foi encontrada positividade em apenas 17 crianças (15,3\%), 14 com vírus respiratório sincicial e as demais ( 3 casos) com vírus influenza. Dezessete pacientes não fizeram o exame $(15,3 \%)$.

Tabela 1 - Antecedentes pessoais dos pacientes

\begin{tabular}{lcc}
\hline Antecedentes & N & \% \\
\hline Prematuridade informada & 26 & 23,4 \\
Peso de nascimento $<2.500 \mathrm{~g}$ & 15 & 13,6 \\
Sibilância prévia & 50 & 45 \\
Hospitalização prévia por sibilância & 31 & 27,9 \\
Oxigenoterapia prévia por mais de 7 dias & 19 & 17,1 \\
Uso de $\mathrm{O}_{2}$ há menos de 30 dias & 19 & 17,1 \\
Uso de ventilação mecânica prévia & 9 & 8,1 \\
\hline
\end{tabular}

$\mathrm{N}$ = número de crianças. 
Quanto às medidas seqüenciais, pôde-se observar que as médias de escores clínicos se mantiveram entre 4 e 5, não tendo havido variação entre os grupos com e sem sibilância prévia. Analisando correlações entre os escores clínicos e os tempos de oxigenoterapia e de internação, os coeficentes de correlação variaram de $-0,007$ a 0,44 , não foram observadas correlações significativas $(p=0,002)$, tanto no grupo geral quanto no grupo sem história de sibilância prévia.

Tomando-se como exemplo o tempo de oxigenoterapia (Figura 1), observa-se uma redução do número de pacientes a cada medida realizada.

Cinco crianças $(4,5 \%)$ evoluíram para necessidade de ventilação mecânica, a maioria $(n=4)$ com história de sibilância prévia.

Os pacientes que estavam no primeiro episódio de sibilância tiveram uma mediana de uso de oxigênio suplementar total de 107 horas. A mediana do tempo de uso de oxigênio até o lactente saturar $95 \%$ em ar ambiente foi de 83 horas e a do tempo de internação foi de 7 dias, neste mesmo grupo. As crianças com história de sibilância prévia tiveram médias semelhantes, exceto pelo tempo de internação, como se pode verificar na Tabela 2. Ocorreram 8 altas hospitalares antes dos pacientes saturarem $95 \% \mathrm{em}$ ar ambiente, motivo pelo qual somente 103 pacientes tiveram medidos o tempo de oxigenoterapia com este desfecho. Em relação ao tempo de internação, observou-se que $47,7 \%$ ficaram hospitalizados sete ou menos dias.

Os TDs a $90 \%$ apresentaram poucas variações nas medianas dos diferentes momentos de medida, como evidenciado na Figura 2. Os TDs a 85\% também não apresentaram um padrão de decréscimo.

Não houve diferença estatística nos grupos sem e com sibilância prévia em relação ao TD90. Não foi possível comparar esses dois grupos em relação ao TD85, pelo número reduzido de pacientes em ambas as categorias.

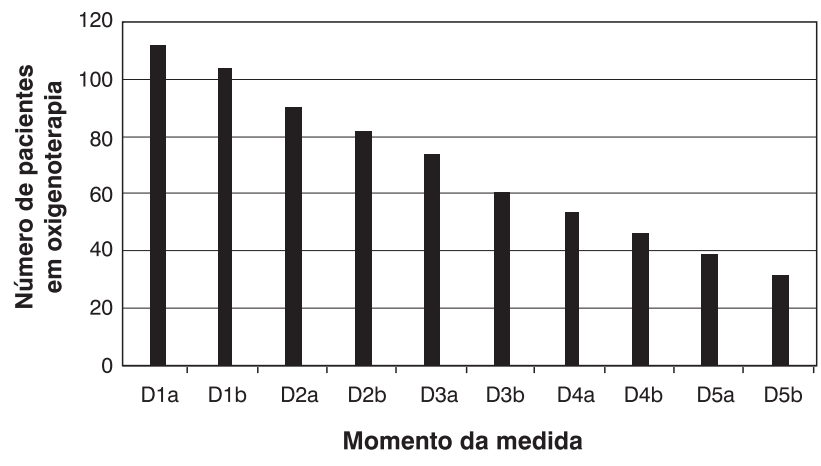

$\mathrm{D} 1 \mathrm{a}=$ primeira medida $\mathrm{D} 1 \mathrm{~b}=$ segunda medida $; \mathrm{D} 2 \mathrm{a}=$ terceira medida; $\mathrm{D} 2 \mathrm{~b}=$ quarta medida; $\mathrm{D} 3 \mathrm{a}=$ quinta medida; $\mathrm{D} 3 \mathrm{~b}=$ sexta medida; $\mathrm{D} 4 \mathrm{a}=$ sétima medida; $\mathrm{D} 4 \mathrm{~b}=$ oitava medida; $\mathrm{D} 5 \mathrm{a}=$ nona medida; $\mathrm{D} 5 \mathrm{~b}=$ décima medida.

Figura 1 - Número de pacientes em oxigenoterapia nos primeiros dias de internação

Não houve correlações significativas entre os tempos de dessaturação (90\% e 85\%) e os desfechos propostos tempo de oxigênio suplementar durante a internação, tempo de oxigenoterapia até atingir saturação maior que 95\% em ar ambiente e tempo de internação, considerando $\mathrm{p}=0,002$. Os coeficientes de correlação entre os TD 90 e os desfechos variaram de $-0,0047$ a 0,83 .

\section{Discussão}

Empiricamente, a medida de TD tem sido realizada na enfermaria de pneumologia do Hospital da Criança Santo Antônio, de Porto Alegre, especialmente em pacientes com uso de oxigenoterapia prolongada. Nenhum estudo até o momento avaliou a relação entre tempo de dessaturação de $\mathrm{O}_{2}$ após a suspensão do oxigênio suplementar e evolução de lactentes hospitalizados por BVA.

Tabela 2 - Oxigenoterapia e tempo de internação dos pacientes

\begin{tabular}{lccccc}
\hline Desfechos & \multicolumn{2}{c}{ Sem sibiância prévia } & \multicolumn{3}{c}{ Com sibilância prévia } \\
& $\mathbf{N}$ & $\begin{array}{c}\text { Mediana } \\
\text { (IIQ) }\end{array}$ & $\mathbf{N}$ & $\begin{array}{c}\text { Mediana } \\
\text { (IIQ) }\end{array}$ & $\mathbf{p}$ \\
\hline $\mathbf{O}_{\mathbf{2}}$ total (h) & 61 & $107(70-160)$ & 50 & $115(79-195)$ & 0,21 \\
O $_{\mathbf{2}}$ para sat 95\% (h) & 56 & $83(55-128)$ & 47 & $79(44-119)$ & 0,75 \\
Internação (dias) & 61 & $7(5-10)$ & 50 & $9,5(5-12)$ & 0,041 \\
\hline
\end{tabular}

$\mathrm{N}=$ número de crianças, $\mathrm{h}=$ dias, $\mathrm{IIQ}=$ intervalo interquartis, $\mathrm{O}_{2}$ total = tempo total de oxigenoterapia durante a internação, $\mathrm{O}_{2}$ para sat $95 \%$ = tempo de oxigenoterapia até a primeira medida de saturação transcutânea de oxigênio da hemoglobina de $95 \%$ em ar ambiente, internação = período em que o paciente esteve internado. 


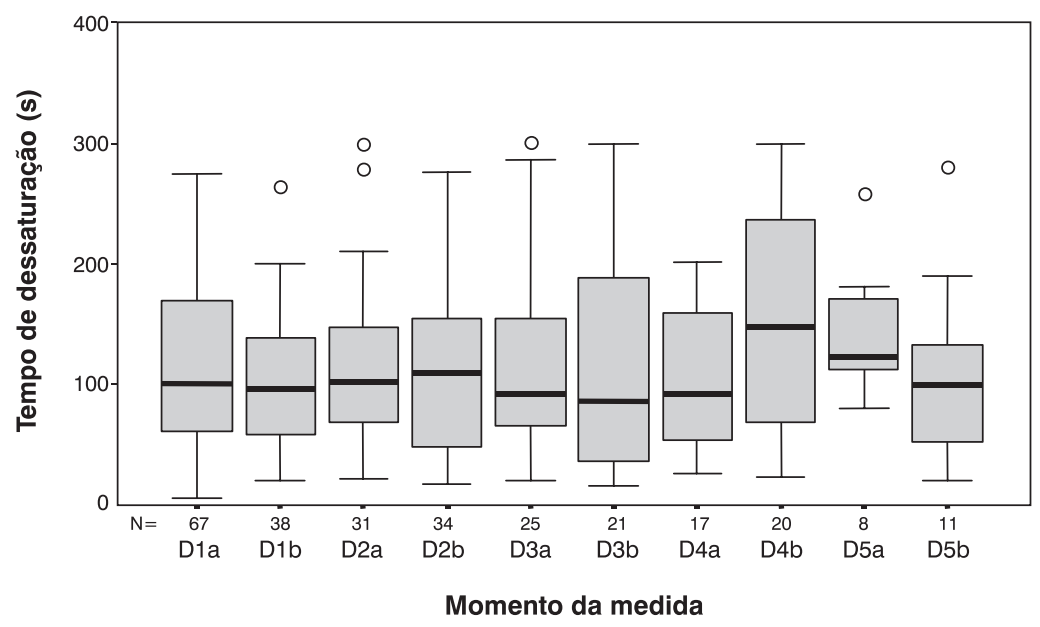
$\mathrm{S}=$ segundos, $\mathrm{D} 1 \mathrm{a}=$ primeira medida, $\mathrm{D} 1 \mathrm{~b}=$ segunda medida, $\mathrm{D} 2 \mathrm{a}=$ terceira medida,
$\mathrm{D} 2 \mathrm{~b}=$ quarta medida, $\mathrm{D} 3 \mathrm{a}=$ quinta medida, $\mathrm{D} 3 \mathrm{~b}=$ sexta medida, $\mathrm{D} 4 \mathrm{a}=$ sétima medida,
$\mathrm{D} 4 \mathrm{~b}=$ oitava medida, $\mathrm{D} 5 \mathrm{a}=$ nona medida, $\mathrm{D} 5 \mathrm{~b}=$ décima medida.

Figura 2 - Tempo de dessaturação a 90\% nos primeiros dias de internação

Foram elegíveis para o estudo 122 crianças e, destas, estudaram-se $96 \%$, com o que se obteve uma amostra representativa de pacientes dentro dos critérios de inclusão propostos. Considerando a história natural da BVA, em que geralmente as alterações mais intensas ocorrem nos primeiros dias de doença ${ }^{7}$, o seguimento parece ter sido suficientemente prolongado para detectar-se a gravidade do evento.

No que se refere à época de internação, ao sexo e à idade, os resultados encontrados foram semelhantes aos do estudo de Fischer ${ }^{16}$, realizado no mesmo hospital. Outros autores obtiveram achados semelhantes ${ }^{9,17-19}$.

Identificou-se prevalência elevada de prematuridade (23\%) e de baixo peso de nascimento (14\%). A associação de BVA e baixo peso ao nascimento foi destacada em estudo anterior ${ }^{16}$ e mereceria maior atenção. Segundo o autor, definir o papel prognóstico ou de risco dessa condição em BVA poderia auxiliar no melhor entendimento de sua morbidade.

As manifestações clínicas que determinaram a internação ocorreram em sua maioria nos três dias anteriores, o que caracteriza o caráter agudo da doença.

O diagnóstico de BVA incluiu critérios já utilizados por outros autores, que reforçam a importância dos aspectos clínicos para a caracterização da doença ${ }^{2,3,20}$. Há autores que questionam a não inclusão de pacientes com episódios recorrentes de sibilância provocada por viroses nos critérios de BVA ${ }^{21,22}$. A dificuldade de diagnóstico em lactentes que apresentam sibilância também se observou neste estu- do, evidenciada nos diversos rótulos diagnósticos utilizados durante a internação: broncoespasmo, displasia broncopulmonar, asma, lactente sibilante e bronquiolopatia pós-viral.

Considerando que o TD de oxigênio na suspensão do oxigênio suplementar tenha possivelmente mecanismos fisiopatológicos semelhantes nos lactentes com BVA e nos com sibilância recorrente (diminuição da via aérea, edema, reação inflamatória), resolveu-se não excluir esse último grupo. Adicionalmente, a análise estatística desse grupo foi realizada separadamente e não evidenciou diferença em relação à população total. Por outro lado, o presente estudo avaliou a condição "dessaturação" em lactentes com episódio agudo de doença de vias aéreas baixas, com o objetivo de estabelecer parâmetros clinicamente úteis. Portanto não parece ser uma limitação importante os pacientes terem ou não diagnóstico preciso de BVA. Como descrito anteriormente, os grupos com BVA e com história de sibilância prévia estudados foram homogêneos na maioria dos aspectos avaliados.

A identificação etiológica viral foi uma das limitações enfrentadas neste estudo, pois ela poderia ter caracterizado melhor a população se a positividade encontrada fosse maior. Vários entraves técnicos possivelmente influenciaram os resultados, já que os exames foram realizados na dependência da rotina do hospital. Como já comentado anteriormente, essa limitação não prejudicou a análise, pois os pacientes estudados tinham doença ventilatória de caráter agudo. 
Quanto à desnutrição, encontrou-se uma frequiência elevada. Essa condição mereceria maior investigação em crianças com BVA, pois poucos estudos a avaliaram especificamente ${ }^{23,24}$. Sabe-se que a resposta imunológica em pacientes desnutridos pode estar alterada e, potencialmente, contribuir para uma evolução desfavorável. Fischer ${ }^{15}$ observou que a desnutrição grave aumenta o risco dos pacientes desenvolverem pior prognóstico (risco relativo igual a 2,0).

O tratamento da BVA tem-se modificado pouco ao longo dos anos e, em muitos casos, a estratégia terapêutica empregada carece de evidências ${ }^{25}$. Uma meta-análise, envolvendo oito ensaios clínicos randomizados, concluiu que os broncodilatadores produziram modesta melhora no escore clínico e por curto período de tempo (a inclusão de pacientes com sibilância prévia poderia ter ocasionado um viés a favor do uso dos broncodilatadores), porém não reduziram o tempo de hospitalização, não melhoraram as medidas de oxigenação e nem diminuíram a necessidade de internação ${ }^{26}$. Martinón-Torres e colaboradores ${ }^{25}$ constataram que, apesar da falta de evidência favorável para o uso de beta-2-agonista na BVA e da recomendação mais aceita de que ele não seja utilizado de forma rotineira, o uso desse tipo de medicamento ainda é amplamente difundido (cerca de $80 \%$ dos pacientes). Todos os pacientes da presente pesquisa utilizaram broncodilatadores inalatórios, geralmente através de nebulização com fenoterol.

Registrou-se alta frequiência de uso de corticosteróides sistêmicos nos pacientes com BVA (20\%), prática não recomendada. Seu emprego, entretanto, foi bem maior nas crianças com história de sibilância prévia $(78 \%)$, o que pode refletir uma tendência de interpretá-las como asmáticas. Alguns estudos encontraram efeitos positivos no uso de corticóides na BVA. Destaca-se uma meta-análise incluindo seis ensaios clínicos randomizados, com um total de 347 pacientes - realizada para determinar se os corticosteróides sistêmicos são eficazes no tratamento da bronquiolite em lactentes hospitalizados. As crianças que receberam corticosteróides tiveram melhora significativa na duração dos sintomas e uma discreta diminuição no tempo de internação, quando comparadas com o grupo que recebeu placebo. Entretanto, limitações dessa análise como ensaios clínicos heterogêneos, duas pesquisas que não excluíram pacientes com sibilância prévia, adoção de escores clínicos diferentes nos estudos e o próprio viés de publicação - permitem que se questionem esses resultados ${ }^{27}$.

Também ocorreu frequiência elevada de emprego de antibioticoterapia sistêmica (47\%). Henderson e Rubin ${ }^{28}$, avaliando crianças internadas por BVA, observaram que cerca de metade dos pacientes receberam antimicrobianos, um terço sem nenhuma evidência documentada de foco infeccioso bacteriano. Além disso, em quase $80 \%$ dos pacientes que receberam antibióticos, seu uso se deu em razão de diagnóstico errôneo de pneumonia bacteriana. Tal quadro reflete o desconforto dos pediatras em atribuir achados radiográficos anormais à presença de patógenos virais na BVA, apesar da evidência na literatura médica de que antibióticos não oferecem benefícios neste contexto.

A mediana do uso de oxigênio em pacientes com BVA e o tempo de oxigenoterapia até saturar $95 \%$ em ar ambiente foram semelhantes aos resultados encontrados por Fischer ${ }^{15}$.

Apesar dos resultados relatados em várias pesquisas envolvendo BVA, na prática clínica ainda se observam diferenças em relação ao tempo de hospitalização entre diversos centros ${ }^{29,30}$. Na maior parte da Europa, lactentes admitidos no hospital recebem alta somente quando estão completamente recuperados. A média de permanência é de oito a nove dias ${ }^{31}$. Na América do Norte, na Austrália, no Reino Unido e na Finlândia, a média de tempo de internação é de quatro dias ${ }^{8}$. Considerando o segmento da população predominantemente atendida no hospital em que foi realizada a pesquisa, o nível socioeconômico pode ter influenciado no tempo de permanência de alguns pacientes.

Em relação ao escore clínico aplicado, observou-se pouca mudança no período estudado, o que sugere pouca variabilidade clínica nos pacientes avaliados. Fischer ${ }^{15}$ encontrou diferenças mais marcantes em sua pesquisa. Um fator que pode estar implicado na homogeneidade dos resultados foram os critérios de seleção adotados. Os pacientes apresentavam quadro clínico de moderada gravidade. A baixa frequiência de pacientes que necessitaram de ventilação mecânica evidencia a não inclusão de pacientes com maior gravidade. A exclusão dos pacientes que não necessitavam de oxigenoterapia e dos que necessitavam de concentrações de oxigênio mais elevadas pode ter contribuído para que a população do estudo fosse semelhante entre si. Tomando-se como exemplo o tempo de oxigenoterapia, observou-se a mesma tendência evidenciada no estudo de Fischer ${ }^{15}$, com redução do número de pacientes por melhora das condições clínicas a cada dia de avaliação, pois não necessitavam mais de oxigênio. Essa questão também pode ter contribuído para a pouca variabilidade clínica dos escores clínicos, já que somente os pacientes em oxigenoterapia foram avaliados. As correlações dos escores clínicos com os desfechos (tempos de oxigenoterapia e de internação) não foram significativas nos pacientes com BVA, provavelmente também em razão dos critérios de seleção.

Assim como ocorreu em relação aos escores clínicos, os TDs encontrados apresentaram poucas variações. É provável que essa pouca variabilidade dos dados esteja relacionada com a questão, já abordada, da homogeneidade da população estudada. Os TDs não apresentaram correlações significativas com os desfechos (tempo de oxigenoterapia, tempo para saturar 95\% em ar ambiente e tempo de internação). A análise de dados que incluíssem os pacientes com maior gravidade, possivelmente, revelaria resultados mais expressivos. 
Outro ponto que poderia, eventualmente, interferir nos resultados foi o uso de dois oxímetros com características diferentes durante a pesquisa. Os equipamentos poderiam apresentar diferenças na resposta à dessaturação, podendo um aparelho ser mais rápido do que o outro. Entretanto, além de não ter sido evidenciada diferença marcante nos resultados dos TDs, parece que essa variabilidade ocorre mais acentuadamente durante hipoxemia mais grave. Trivedi e colaboradores ${ }^{32}$ compararam a eficácia e os tempos de respostas de dessaturação e ressaturação de sete oxímetros de pulso durante a indução de hipoxemia, em oito voluntários hígidos $\left(\mathrm{FiO}_{2}\right.$ a $\left.10 \%\right)$. A maioria dos modelos testados teve um bom desempenho, mas foram ineficazes quando a saturação de oxigênio atingia aproximadamente $75 \%$ ou menos. A maior diferença no tempo de resposta de dessaturação entre o mais rápido e o mais lento oxímetro foi de 13 a 29 segundos. Não houve diferença estatística entre os oxímetros durante a fase de ressaturação.

Pode-se perceber, pelos dados apresentados, que a idéia de usar os TDs de oxigênio como elementos auxiliares na avaliação de pacientes em oxigenoterapia não pode ser rechaçada nem valorizada. É possível que, avaliando-se pacientes com maiores diferenças clínicas entre si, essas aferições (TDs) possam mostrar-se importantes, sendo necessários novos estudos para avaliar melhor a utilidade do TD no lactente com doença pulmonar obstrutiva aguda. É importante ressaltar que a saturação transcutânea de oxigênio continua sendo um parâmetro funcional valioso no acompanhamento clínico da BVA, especialmente em pacientes hospitalizados.

\section{Referências bibliográficas}

1. Albernaz E. Epidemiologia da hospitalização por bronquiolite aguda no período pós-natal em Pelotas, RS [dissertação]. Pelotas: Universidade Federal de Pelotas; 1997.

2. Court SDM. The definition of acute respiratory illnesses in children. Postgrad Med J 1973;49:771-6.

3. McConnochie KM. Bronchiolitis - what's in the name. Am J Dis Child 1983;137:11-3.

4. Tissing WJE, van Steensel-Moll HA, Offringa M. Risk factors for mechanical ventilation in respiratory syncytial virus infection. Eur J Pediatr 1993;152:125-7.

5. Darville T, Yamauchi T. Respiratory syncytial virus. Pediatr Rev 1998;19:55-61.

6. Kopelman BI, Miyoshi MH. Infecção pelo vírus sincicial respiratório. Pediatria Moderna 1999;35:233-39.

7. Whol MEB, Chernick V. Bronchiolitis, state of art. Am Rev Respir Dis 1978;118:759-81.

8. Simoes EAF. Respiratory syncytial virus infection. Lancet 1999;354:847-52.

9. La Via WV, Marks MI, Stutman HR. Respiratory syncytial virus puzzle: clinical, features, pathophysiology, treatment, and prevention. J Pediatr 1992;121:503-10.

10. Everard ML, Milner AD. The respiratory syncytial virus and its role in acute bronchiolitis. Eur J Pediatr 1992;151:638-51.
11. Reynolds EOR, Cook CD. The treatment of bronchiolitis. J Pediatr 1963;6:1205-7.

12. Pio A. Appropriate technology for the administration of oxygen to children at district hospitals in developing countries. Int J Tuberc Lung Dis 2001;5:493-5.

13. Tremper KK, Barker SJ. Pulse oximetry. Anesthesiology 1989;70:98-108.

14. Barker SJ. Standardization of the testing of pulse oximeter performance. Anesth Analg 2002;94 Supl 1:17-20.

15. Fischer GB. Fatores prognósticos para bronquiolite viral aguda [tese]. Porto Alegre: Universidade Federal do Rio Grande do Sul; 1994.

16. Goulart EMA. A avaliação nutricional infantil no software EPI INFO (versão 6.0), considerando-se a abordagem coletiva e individual, o grau e tipo da desnutrição. J Pediatr (Rio J) 1997;73:225-30.

17. Weber MW, Mulholland EK, Greenwood BM. Respiratory syncytial virus infection in tropical and developing countries. Trop Med Int Health 1998;3:268-80.

18. Vieira SE, Stewien KE, Queiroz DAO, Durigon EL, Török TJ, Anderson LJ, et al. Clinical patterns and seasonal trends in respiratory syncytial virus hospitalizations in São Paulo, Brazil. Rev Inst Med Trop Sao Paulo 2001;43:125-31.

19. Chanock RM, Kim HW, Vargosko AJ, Deleva A, Johnson KM, Cumming C, et al. Respiratory Syncytial Virus. Virus recovery and other observations during 1960 outbreak of bronchiolitis, pneumonia, and minor respiratory diseases in children. JAMA 1961;176:647-53.

20. Sant'Anna CC, D'Elia C. Bronquiolite. In: Benguigui Y, Antuñano FJL, Schmunis G, Yunes J. Infecções respiratórias em crianças. Washington: OPAS/OMS; 1998. p. 263-81.

21. Welliver JR, Welliver RC. Bronchiolitis. Pediatr Rev 1993;14: 134-9.

22. D’Elia C, Barbosa MCM. Abordagem na disfunção respiratória aguda. J Pediatr (Rio J) 1999;75 Supl 2:168-76.

23. McIntosh K. Pathogenesis of severe acute respiratory infections in developing world: respiratory syncytial virus and parainfluenzae viroses. Rev Infect Dis 1991;13 Supl 6:492-500.

24. Banerji A, Bell A, Mills EL, McDonalds J, Subbarao K, Stark G, et al. Lower respiratory tract infections in Inuit infants on Baffin Island. CMAJ 2001;164:1847-50.

25. Martinón-Torres F, Núñez AR, Sánchez JMM. Bronquiolitis aguda: evaluación del tratamiento basada en la evidencia. An Esp Pediatr 2001;55:345-54.

26. Kellner JD, Ohlsson A, Gadomski AM, Wang EEL. Bronchodilators for bronchiolitis. Cochrane Database Syst Rev. 2000;(2):CD001266.

27. Garrison MM, Christakis DA, Harvey EH, Cummings P, Davis RL. Systemic corticosteroids in infant bronchiolitis: a metaanalysis [periódico eletrônico]. Pediatrics 2000;105:e44. Disponível: URL: http://www.pediatrics.org//content/full/105/4/ e44. Acessado: 2 de dezembro de 2002.

28. Henderson M, Rubin E. Misuse of antimicrobials in children with asthma and bronchiolitis: a review. Ped Inf Dis J 2001;20:214-5.

29. Wang EE, Law BJ, Boucher FD, Stephens D, Robinson JL, Dobson S, et al. Pediatric Investigators Collaborative Network on Infections in Canada (PICNIC) study of admission and management variation in patients hospitalized with respiratory syncytial viral lower respiratory tract infection. J Pediatr 1996;129:390-5.

30. Brand PLP, Vaessen-Verberne AAPH. Differences in management of bronchiolitis between hospitals in The Netherlands. Eur J Pediatr 2000;159:343-7. 
31. Behrendt CE, Decker MD, Burch DJ, Watson PH. International variation in the management of infants hospitalized with respiratory syncytial virus. International RSV Study Group. Eur J Pediatr 1998;157:215-20.

32. Trivedi NS, Ghouri AF, Shah NK, Lai E, Barker SJ. Effects of motion, ambient light, and hypoperfusion on pulse oximeter function. J Clin Anesth 1997;9:179-83.
Endereço para correspondência:

Dra. Fernanda Menezes Rubin

Rua Corcovado, 122/502

CEP 90540-100 - Porto Alegre - RS

Tel.: (51) 3343.6872

E-mail: frubin@terra.com.br 